\title{
Hybrid Fuzzy-Linear Programming with Shadowed Fuzzy Numbers
}

\author{
Mohamed A. H. \\ El_Hawy \\ Faculty of Computer \\ and Information, \\ Cairo University, \\ Giza- Egypt
}

\author{
Khaled T. Wassif \\ Faculty of Computer \\ and Information, \\ Cairo University, \\ Giza- Egypt
}

\author{
Hesham A. Hefny \\ Institute of Statistical \\ Studies and Research, \\ Cairo University \\ Giza- Egypt
}

\author{
Hesham A. Hassan \\ Faculty of Computer \\ and Information, \\ Cairo University, \\ Giza- Egypt
}

\begin{abstract}
Linear programming problem in an environment that includes different types of uncertainties represents real-world situations. In such situations, different forms of uncertain data parameters are commonly found in that problem. Fuzzy sets and their extensions are important tools of representing vague information. For decades, a lot of approaches are developed to solve fuzzy-linear programming problems. The existence of hybrid types of uncertainties in the fuzzy-linear programming problem imposes a real challenge to solve it. There is a need for introducing an efficient methodology to transform different types of uncertainties into a unified form. This paper introduces a new approach to solve hybrid fuzzylinear programming using an improved version of shadowed fuzzy numbers (SFNs). SFNs are useful transformation tool for different types of uncertainties. They have the advantage of preserving the characteristics of uncertainty for different types of fuzzy sets used in the problem.
\end{abstract}

\section{Keywords}

Shadowed sets , Fuzzy numbers, Intuitionistic fuzzy numbers , Non-specificity measure , Entropy measure , Fuzzy linear programming

\section{INTRODUCTION}

Vague information are represented and processed by using fuzzy sets and their extensions. The fuzzy membership function contains two types of uncertainties, namely: fuzziness and nonspecificity [1]. Fuzziness and nonspecificity are related to the imprecise boundaries and the cardinality of fuzzy sets respectively $[1,2]$. Intuitionistic fuzzy sets (IFSs) are characterized by two functions (membership and nonmembership). It's more flexible than fuzzy sets. Entropy and nonspecificity represent basic types of uncertainty for IFSs [3, 4]. The shadowed sets are firstly proposed by Pedrycz to approximate fuzzy sets by using a three-valued constructs, namely $(0,[0,1], 1)[5]$. Pedrycz's method was motivated by the principle of uncertainty localization in which an optimization problem is formulated to redistribute the uncertainty content of a fuzzy set into the above three-valued construct [6]. The important properties of shadowed sets are preserving characteristics of uncertainty of fuzzy set and simplifying computations complexity.

Three areas are formed the shadowed set. Excluded area for the elements almost not included in set, core area for the elements that almost certainly belonging to it and shadow area or uncertain area for the elements approximately belong to it. The authors developed an improved shadowed fuzzy numbers (SFNs) that induced form fuzzy numbers and intuitionistic fuzzy numbers $[7,8]$. A new approach is considered to be more accurate as it preserves more than one type of uncertainty. Also, it is appropriate for inducing shadowed sets from higher types of fuzzy sets. Over the past several decades linear programming models have been primarily developed in a crisp environment [1]. After fuzzy decision making is proposed by Bellman and Zadeh [9], many researchers developed this concept for solving fuzzy linear programming problems. In the literature $[1,10]$, the most of the fuzzy linear programming models can be classified to three categories. First, fuzziness related to the objective function coefficients and precise constraints. Second, a precise objective function coefficients and fuzzy constraints coefficients and right hand side values of the constraints. Third, a fuzzy objective function coefficients, fuzzy constraints coefficients and fuzzy right hand side of constraints. Intuitionistic fuzzy- linear programming is proposed by Angelov[11]. Different approaches are developed to solve Intuitionistic fuzzy- linear programming [11], [12], [13], [14]. In all previous mentioned approaches, some or all parameters of fuzzy-linear programming problem is considered from one type of fuzzy numbers and no explicit approach is proposed to solve the problem in the case of hybrid types of them. In this paper, the authors develop a new approach to solve hybrid fuzzylinear programming which includes different uncertain types of parameters. The hybrid data include exact numbers, type-1 fuzzy numbers and intuitionistic fuzzy numbers. The new approach is based on the use of the shadowed fuzzy numbers. The reset of this paper is organized as follows: Section 2 presents an introduction about fuzzy numbers (FNs). It reviews intuitionistic fuzzy sets (IFSs) and an intuitionistic fuzzy numbers (IFNs). Also, this section introduces shadowed sets. Section 3 defines shadowed fuzzy number (SFN) and the new approaches to induce it from FN and IFN and define basic operations for SFNs. Section 4 introduces to proposed steps for solving hybrid fuzzy-linear programming problems. Section 5 provides illustrative examples to explain a new approach. Finally, conclusions are discussed in section 6 .

\section{PRELIMINARIES}

In this section, some basic definitions and properties relevant to the present work are mentioned for fuzzy numbers (FN), intuitionistic fuzzy numbers (IFNs) and shadowed sets.

\subsection{Fuzzy Numbers (FNs)}

Some types of fuzzy sets $\widetilde{\boldsymbol{F}}$ are defined on set $\mathbb{R}$ of real numbers and used to approximate uncertainty concept about numbers or intervals. Such types are called fuzzy numbers or fuzzy intervals and have the following conditions $[1,15]$

- $\widetilde{\boldsymbol{F}}$ is normal, i.e. at least one element $x_{i}$ such that $\mu\left(x_{i}\right)=1$. 
- $\widetilde{\boldsymbol{F}}$ is convex such that $\widetilde{\boldsymbol{F}}(\boldsymbol{\lambda} \boldsymbol{x}+(\mathbf{1}-\boldsymbol{\lambda}) \boldsymbol{y}) \geq$ $\min (\widetilde{\boldsymbol{F}}(\boldsymbol{x}), \widetilde{\boldsymbol{F}}(\boldsymbol{y})) \forall \boldsymbol{x}, \boldsymbol{y} \in \mathrm{U}$ and $\lambda \in[0,1]$ where $\mathbf{U}$ is a universe of discourse.

- $\quad$ The support of $\widetilde{\boldsymbol{F}}$ is bounded.

The membership function of the real fuzzy number $\widetilde{\boldsymbol{F}}$ is defined by [16]

$$
\mu_{\widetilde{F}}(x)=\left\{\begin{array}{lr}
l_{\widetilde{F}}(x) & \text { if } a \leq x \leq b, \\
1 & \text { if } b \leq x \leq c, \\
r_{\widetilde{F}}(x) & \text { if } c \leq x \leq d, \\
0 & \text { otherwise }
\end{array}\right.
$$

where $\boldsymbol{l}_{\widetilde{\boldsymbol{F}}}$ and $\boldsymbol{r}_{\widetilde{\boldsymbol{F}}}$ are two continuous increasing and decreasing functions for left and right side of fuzzy number and a, b, c, d are real numbers.

\subsection{Intuitionistic fuzzy numbers (IFNs)}

An intuitionistic fuzzy number is an intuitionistic fuzzy set that defines on real numbers scale as in Fig. 1 and is defined as $[17,18]$

$$
\mathbf{A}=\left\{<x, \mu_{\mathbf{A}}(\mathbf{x}), \mathbf{v}_{\mathbf{A}}(\mathbf{x})>\mid \mathbf{x} \in \mathbb{R}\right\},
$$

where $\boldsymbol{\mu}_{\boldsymbol{A}}(\boldsymbol{x}): \boldsymbol{X} \rightarrow[\mathbf{0 , 1}]$ is membership function, $\boldsymbol{\nu}_{\boldsymbol{A}}(\boldsymbol{x})$ $\boldsymbol{X} \rightarrow[\mathbf{0}, \mathbf{1}]$ is non-membership function, such that $[3,4]$ :

$$
\mathbf{0} \leq \mu_{A}(x)+v_{A}(x) \leq 1 .
$$

Let

$$
\pi_{A}=1-\mu_{A}(x)-v_{A}(x),
$$

where $\boldsymbol{\pi}_{\boldsymbol{A}}$ is called the intuitionistic index or hesitancy degree of $\boldsymbol{x}$ in $\boldsymbol{A}$. The IFN $A$ satisfy the following conditions:

1. A is normal, i.e. at least two points $\boldsymbol{x}_{\mathbf{0}}, \boldsymbol{x}_{\mathbf{1}}$ belong to A such that $\mu_{A}\left(x_{0}\right)=1, v_{A}\left(x_{1}\right)=1$.

2. A is convex, i.e. $\boldsymbol{\mu}_{\boldsymbol{A}}$ is fuzzy convex and $\boldsymbol{v}_{\boldsymbol{A}}$ is fuzzy concave.

3. $\boldsymbol{\mu}_{\boldsymbol{A}}$ is upper semicontinuous and $\boldsymbol{v}_{\boldsymbol{A}}$ is lower semicontinuous.

4. $\operatorname{support}(A)=\left\{\boldsymbol{x} \in \boldsymbol{X} \mid \boldsymbol{v}_{\boldsymbol{A}}(\boldsymbol{x})<1\right\}$ is bounded.

Some type like triangular intuitionistic fuzzy set as in Fig.1 and identified as [17]

$$
T I F S=<(a, b, c),(\bar{a}, b, \bar{c}) ; M_{A}, V_{A}>,
$$

where $(\mathbf{a}, \mathbf{b}, \mathbf{c}),(\overline{\mathbf{a}}, \mathbf{b}, \overline{\mathbf{c}})$ are basic values of TIFS , ... largest membership value and $\boldsymbol{V}_{\boldsymbol{A}}$ is lowest non-memberaship value.

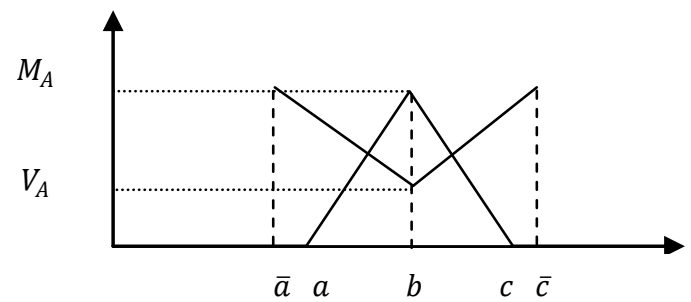

Fig. 1. Triangular intuitionistic fuzzy number

\subsection{Shadowed sets}

Shadowed sets are induced from fuzzy sets which preserving the essence of fuzzy sets and reducing the computational costs. Shadowed set $\mathrm{S}$ is defined as $[5,19]$

$$
S: X \rightarrow\{0,1,[0,1]\},
$$

The membership function of fuzzy set is transformed to the form is similar to three-valued logic. For preserving the uncertainty modeled by fuzzy sets, the process of creating shadowed set start by selecting the threshold $\boldsymbol{\alpha} \in(\mathbf{0}, \mathbf{0 . 5})$. Selecting $\alpha$ depend on achieve a balance of uncertainty between three regions. The shadow region $\boldsymbol{r}_{3}$, the region $\boldsymbol{r}_{2}$ which represent the uncertainty lost due to elevating membership values to 1 and the region $\boldsymbol{r}_{1}$ where the membership values is reduced to 0 . This balance is defined as the following equation [5].

$$
\boldsymbol{u}\left(\boldsymbol{r}_{1}\right)+\boldsymbol{u}\left(\boldsymbol{r}_{2}\right)=\boldsymbol{u}\left(\boldsymbol{r}_{3}\right),
$$

where $\mathbf{u}$ is uncertainty of regions $\boldsymbol{r}_{\mathbf{1}}, \boldsymbol{r}_{\mathbf{2}}$ and $\boldsymbol{r}_{\mathbf{3}}$. Pedrycz proposed minimization of performance index $\boldsymbol{V}_{\boldsymbol{\alpha}}$ for the threshold $\alpha$ as

$$
\boldsymbol{V}_{\alpha}=\left|\boldsymbol{u}\left(\boldsymbol{r}_{1}\right)+\boldsymbol{u}\left(\boldsymbol{r}_{2}\right)-\boldsymbol{u}\left(\boldsymbol{r}_{3}\right)\right|
$$

After getting an optimal $\alpha$, three regions can be induceu irom fuzzy set to represent the core, shadow and exclusion areas as illustrated in Fig. 2. [19].

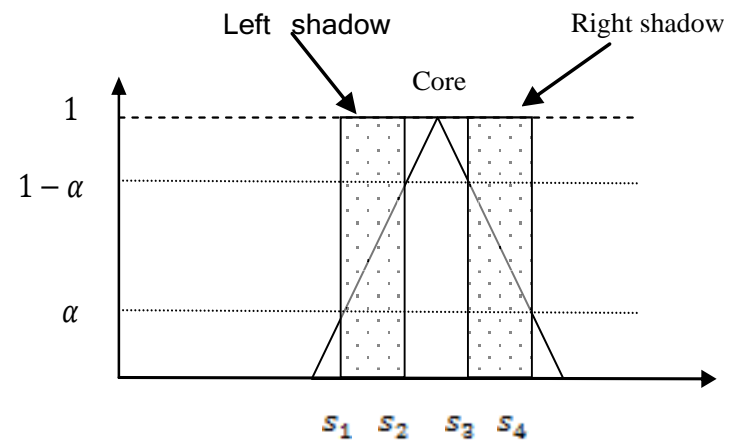

Fig. 2. Shadowed sets induced from triangular fuzzy number

\section{SHADOWED FUZZY NUMBERS}

Shadowed fuzzy number (SFN) is defined as a shadowed set induced from fuzzy number [20]. The authors proposed a new method to induce shadowed fuzzy numbers from fuzzy numbers by constructing core interval and fuzziness intervals [7]. The core interval induces using Hartley non-specificity measure [1] as the following equation

$$
A_{R}(\alpha)-A_{L}(\alpha)+1=2^{H_{A}},
$$

where $\mathbf{H}_{\mathbf{A}}$ is Hartley non-specificity value of fuzzy $\mathbf{A}_{\mathbf{L}}(\boldsymbol{\alpha}), \mathbf{A}_{\mathbf{R}}(\boldsymbol{\alpha})$ are left and right $\alpha$-cut of fuzzy set $f \ldots \ldots \ldots$ interval is deduced by using the $\alpha$-core at level 1 . The fuzziness intervals are based on the use of cardinality of left and right fuzziness sets as.

$$
\begin{gathered}
w_{L}=\sum_{x_{L}} f_{A}\left(x_{L}\right) \\
w_{R}=\sum_{x_{R}} f_{A}\left(x_{R}\right)
\end{gathered}
$$

where $\boldsymbol{w}_{L}, \boldsymbol{w}_{R}$ are width of left and right of iuzziness intervals. $\boldsymbol{x}_{L}$ and $\boldsymbol{x}_{R}$ are the left support and right support of fuzzy numbers from core value. $\boldsymbol{f}_{A}\left(\boldsymbol{x}_{\boldsymbol{L}}\right), \boldsymbol{f}_{\boldsymbol{A}}\left(\boldsymbol{x}_{\boldsymbol{R}}\right)$ are fuzziness sets of fuzzy number which proposed by Tahayori [21] that defined as the following. 


$$
f_{A}=(x, f u z z(x)),
$$

$$
f u z z(x)=1-\left|2 \mu_{A}(x)-1\right| .
$$

Then shadowed fuzzy number (SFN) is constructed oy using core interval and fuzziness intervals as in Fig. 3.
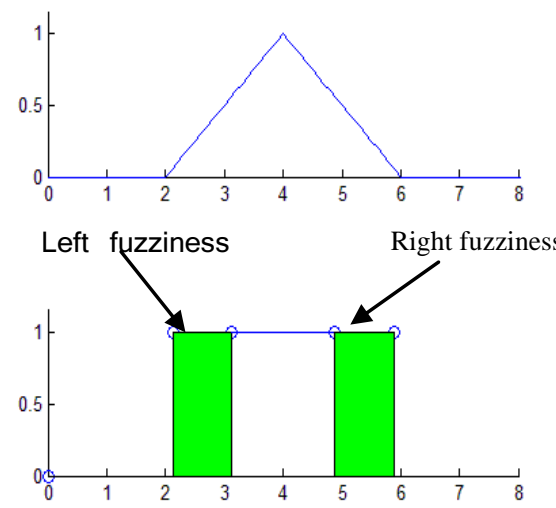

Fig. 3. SFN for triangular fuzzy number

\subsection{Shadowed intuitionistic fuzzy numbers (SIFN)}

Shadowed intuitionistic fuzzy numbers (SIFN) is extended version of SFNs in the case of normal intuitionistic fuzzy numbers IFNs [8]. The proposed approach is developed to induce the core interval of IFN as the following steps. The IFN is transformed to interval fuzzy number as [8]

$$
\mathbf{A}(\boldsymbol{x})=[\boldsymbol{\mu}(\boldsymbol{x}), \mathbf{1}-\boldsymbol{v}(\boldsymbol{x})]
$$

where $\boldsymbol{\mu}(\boldsymbol{x})$ is a lower membership function and $\mathbf{1}-\boldsymbol{v}(\boldsymbol{x})$ is an upper membership function. Hartley non-specificity value of IFN is obtained as the following equation

$$
H_{\text {avg }}(A)=\frac{H(\mu(x))+H(1-v(x))}{2}
$$

where $\boldsymbol{H}_{\boldsymbol{a v g}}$ is the average of non-specificity measure, $\boldsymbol{H}(\boldsymbol{\mu}(\boldsymbol{x}))$ and $\boldsymbol{H}(\mathbf{1}-\boldsymbol{v}(\boldsymbol{x}))$ are the Hartley non-specificity measure of membership function and the complement of nonmembership functions. Then $\boldsymbol{H}_{\boldsymbol{a v g}}$ is used to induce the $\alpha$ cores for lower and upper membership functions of intuitionistic fuzzy number (IFN) as the following equations.

$$
\begin{aligned}
& A_{r}^{L}(\alpha)-A_{l}^{L}(\alpha)+1=2^{H_{a v g}^{L}} \\
& A_{r}^{U}(\alpha)-A_{l}^{U}(\alpha)+1=2^{H_{a v g}^{U}}
\end{aligned}
$$

where $A_{l}^{L}(\alpha), A_{r}^{L}(\alpha), A_{l}^{U}(\alpha)$ and $A_{r}^{U}(\alpha)$ are left and right $\alpha$ cut equations for lower and upper membership functions respectively for IFN A. Then $\boldsymbol{\alpha}_{L}$ and $\boldsymbol{\alpha}_{U}$ are induced and using them in previous left and right $\alpha$-cut equations to get the core intervals $\left[\boldsymbol{c}_{l}^{L}, \boldsymbol{c}_{r}^{L}\right]$ and $\left[\boldsymbol{c}_{l}^{U}, \boldsymbol{c}_{r}^{U}\right]$.

In the case of symmetric membership and non-membership functions, two core intervals are equal. In the case of nonsymmetric, the average of two intervals is defined as the following.

$\left[c_{l}, c_{r}\right]=\left[\frac{\left(c_{l}^{L}+c_{l}^{U}\right)}{2}, \frac{\left(c_{r}^{L}+c_{r}^{U}\right)}{2}\right]$
The shadow areas are induced by using entropy intervals. The entropy is used to estimate the fuzziness of IFNs. The entropy sets is proposed to measures the fuzziness of IFNs based on Xia Liang method [22]. It is defined as the following.

$$
\begin{gathered}
E(A)=(x, \operatorname{ent}(x)) \\
\operatorname{ent}(x)=\frac{1-\left|\mu_{A}(x)-v_{A}(x)\right|+\pi_{A}(x)}{1+\pi_{A}(x)}
\end{gathered}
$$

where ent $(\boldsymbol{x})$ is entropy measure based on Xia Liang method [22] and $\boldsymbol{\mu}_{\boldsymbol{A}}, \boldsymbol{v}_{\boldsymbol{A}}$ and $\boldsymbol{\pi}_{\boldsymbol{A}}$ are membership, non-membership and hesitancy degree functions for every element belong to IFN. The cardinality of left and right entropy sets to get the wide of entropy intervals as the following

$$
\begin{aligned}
& w_{L}=\sum_{x_{L}} E(A) \\
& w_{R}=\sum_{x_{R}} E(A)
\end{aligned}
$$

where $\boldsymbol{w}_{L}$ and $\boldsymbol{w}_{R}$ are the wide of left and right entropy intervals and $\boldsymbol{E}(\boldsymbol{A})$ is entropy set for IFN. These intervals represent shadow areas of shadowed intuitionistic fuzzy numbers (SIFN) and start from left and right end points of core interval. The core interval and entropy intervals are used to composition the shadowed intuitionistic fuzzy numbers (SIFN).

\subsection{Shadowed fuzzy numbers operations}

For applying shadowed numbers in decision making applications, the authors defined the basic arithmetic operations between SFNs as the following [23]

Let $\mathbf{S H}_{1}=\left(\mathbf{S}_{1}^{1}, \mathbf{S}_{2}^{1}, \mathbf{S}_{3}^{1}, \mathbf{S}_{4}^{1}\right), \mathbf{S H}_{2}=\left(\mathbf{S}_{1}^{2}, \mathbf{S}_{2}^{2}, \mathbf{S}_{3}^{2}, \mathbf{S}_{4}^{2}\right)$ are two shadowed fuzzy numbers (SFNs).

\section{- Addition}

The addition operation of the two SFNs is defined as

$S_{1}+S_{2}=\left(S_{1}^{1}+S_{1}^{2}, S_{2}^{1}+S_{2}^{2}, S_{3}^{1}+S_{3}^{2}, S_{4}^{1}+S_{4}^{2}\right)$

\section{- Subtraction}

The subtraction operation of the two SFNs where $\mathbf{S H}_{\mathbf{1}} \geq \mathbf{S H}_{\mathbf{2}}$ is defined as

$$
S_{1}-S H_{2}=\left(S_{1}^{1}-S_{4}^{2}, S_{2}^{1}-S_{3}^{2}, S_{3}^{1}-S_{2}^{2}, S_{4}^{1}-S_{1}^{2}\right)
$$

- Multiplication

The multiplication operation of the two SFNs where $\mathbf{S H}_{\mathbf{1}}$ and $\mathbf{S H}_{\mathbf{2}}$ are induced from two positive fuzzy numbers is defined as

$\mathrm{SH}_{1} \times \mathrm{SH}_{2}=\left(S_{1}^{1} \times S_{1}^{2}, S_{2}^{1} \times S_{2}^{2}, S_{3}^{1} \times S_{3}^{2}, S_{4}^{1} \times S_{4}^{2}\right)$

\section{- Division}

The division operation between the two SFNs $\left(\mathbf{S H}_{\mathbf{1}}, \mathbf{S H}_{\mathbf{2}}\right)$, where $S_{1}^{2} \neq 0, S_{2}^{2} \neq 0, S_{3}^{2} \neq 0$ and $S_{4}^{2} \neq 0$ and they induced from two positive fuzzy numbers is defined as

$$
\mathrm{SH}_{1} / \mathbf{S H}_{2}=\left(\mathbf{S}_{1}^{1} / \mathbf{S}_{4}^{2}, \mathbf{S}_{2}^{1} / \mathbf{S}_{3}^{2}, S_{3}^{1} / S_{2}^{2}, S_{4}^{1} / S_{1}^{2}\right)
$$




\section{HYBRID FUZZY-LINEAR PROGRAMMING PROBLEM}

The general fuzzy-linear programming is formulated as [1]

$$
\begin{array}{lc}
\max & \sum_{j=1}^{n} \tilde{\boldsymbol{c}}_{j} \boldsymbol{x}_{j} \\
\text { subject to } & \sum_{j=1}^{n} \widetilde{A}_{i j} \boldsymbol{x}_{j} \leq \widetilde{\boldsymbol{B}}_{\boldsymbol{i}} \\
\text { where } & \boldsymbol{x}_{\boldsymbol{j}} \geq \mathbf{0} \\
& \boldsymbol{i} \in \mathbb{N}, \boldsymbol{i}=\mathbf{1}, \ldots \ldots, \boldsymbol{m}, \\
& \boldsymbol{j} \in \mathbb{N}, \boldsymbol{j}=\mathbf{1}, \ldots \ldots, \boldsymbol{n},
\end{array}
$$

where $\widetilde{\boldsymbol{A}}_{\boldsymbol{i} \boldsymbol{j}}, \widetilde{\boldsymbol{B}}_{\boldsymbol{i}}$ and $\tilde{\boldsymbol{c}}_{\boldsymbol{j}}$ are fuzzy numbers and $\boldsymbol{x}_{\boldsymbol{j}}$ is a fuzzy variable. The $\leq$ is an inequality relation between fuzzy numbers. The arithmetic operations used in FLP model is fuzzy arithmetic operations. Three important cases are distinguished in the literature for modeling FLP. The first case is a precise objective function coefficients and fuzzy constraints coefficients and right hand side values of the constraints. The second case is a fuzzy objective function coefficients, fuzzy constraints coefficients and fuzzy right hand side of constraints. The Third case is a fuzzy objective function coefficients and precise constraints. Many approaches are developed to solve FLPPs [10].

In some situations, the FLP in the form of a hybrid fuzzylinear programming problem (HFLPP) that has hybrid uncertain types about parameters. In such a situation the parameters of linear programming problems may be represented as different types of fuzzy numbers.

In all of the works in [1, $10-14]$, the FLP problems have been studied in which some or all parts of the problem were assumed to be one type of fuzzy numbers (not hybrid types).

In the following subsections, the authors will solve HFLPP in three different cases as mentioned in this section.

\subsection{Precise objective function coefficients and fuzzy constraints}

Let us consider the following fuzzy linear programming problem with $\mathrm{m}$ constraints and $\mathrm{n}$ variables may be formulated as follows:

$\max$

$$
\sum_{j=1}^{n} c_{j} x_{j}
$$

subject to

$$
\begin{aligned}
& \sum_{j=1}^{n} \widetilde{\boldsymbol{k}}_{i j} x_{j} \leq \tilde{\boldsymbol{t}}_{i} \\
& \sum_{j=1}^{n} \widetilde{\boldsymbol{a}}_{i j} \boldsymbol{x}_{j} \leq \widetilde{\boldsymbol{b}}_{\boldsymbol{i}}
\end{aligned}
$$

$$
x_{j} \geq 0
$$

where

$$
\begin{gathered}
i \in \mathbb{N}, i=1, \ldots \ldots, m \\
j \in \mathbb{N}, j=1, \ldots \ldots, n
\end{gathered}
$$

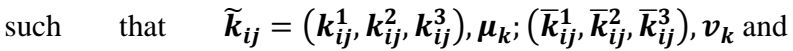
$\tilde{\boldsymbol{t}}_{\boldsymbol{i}}=\left(\boldsymbol{t}_{\boldsymbol{i}}^{\mathbf{1}}, \boldsymbol{t}_{\boldsymbol{i}}^{\mathbf{2}}, \boldsymbol{t}_{\boldsymbol{i}}^{\mathbf{3}}\right), \boldsymbol{\mu}_{\boldsymbol{t}} ;\left(\overline{\boldsymbol{t}}_{\boldsymbol{i}}^{\mathbf{1}}, \overline{\boldsymbol{t}}_{\boldsymbol{i}}^{2}, \overline{\boldsymbol{t}}_{\boldsymbol{i}}^{\mathbf{3}}\right), \boldsymbol{v}_{\boldsymbol{t}} \quad$ are triangular intuitionistic fuzzy numbers TIFNs and $\boldsymbol{\mu}, \boldsymbol{v}$ are membership and and non-membership functions respectively. $\widetilde{\boldsymbol{a}}_{\boldsymbol{i j}}=$ $\left(\boldsymbol{a}_{i j}^{\mathbf{1}}, \boldsymbol{a}_{i j}^{\mathbf{2}}, \boldsymbol{a}_{i j}^{\mathbf{3}}\right), \widetilde{\boldsymbol{b}}_{\boldsymbol{i}}=\left(\boldsymbol{b}_{\boldsymbol{i}}^{\mathbf{1}}, \boldsymbol{b}_{\boldsymbol{i}}^{\mathbf{2}}, \boldsymbol{b}_{\boldsymbol{i}}^{\mathbf{3}}\right)$ are triangular fuzzy numbers TFNs. We use the following steps to solve this HFLPP problem.

Step 1 : The TIFNs $\widetilde{\boldsymbol{k}}_{\boldsymbol{i} \boldsymbol{j}}$ and $\tilde{\boldsymbol{t}}_{\boldsymbol{i}}$ transform to SFNs using equations $(15-23)$ where

$\operatorname{sh}\left(\widetilde{k}_{i j}\right)=\left(s_{k_{i j}}^{1}, s_{k_{i j}}^{2}, s_{k_{i j}}^{3}, s_{k_{i j}}^{4}\right), \operatorname{sh}\left(\tilde{t}_{i}\right)=\left(s_{t_{i}}^{1}, s_{t_{i}}^{2}, s_{t_{i}}^{3}, s_{t_{i}}^{4}\right)$.

Step 2 : The TFNs $\widetilde{\boldsymbol{a}}_{\boldsymbol{i} \boldsymbol{j}}$ and $\widetilde{\boldsymbol{b}}_{\boldsymbol{i}}$ transform to SFNs using equations $(10-14)$ where

$\operatorname{sh}\left(\widetilde{a}_{i j}\right)=\left(s_{a_{i j}}^{1} s_{a_{i j}}^{2}, s_{a_{i j}}^{3}, s_{a_{i j}}^{4}\right), \operatorname{sh}\left(\widetilde{b}_{i}\right)=\left(s_{b_{i}}^{1}, s_{b_{i}}^{2}, s_{b_{i}}^{3}, s_{b_{i}}^{4}\right)$

Step 3 : The problem is reformulated as

$$
\begin{aligned}
& \max \\
& \sum_{j=1}^{n} c_{j} x_{j} \\
& \sum_{j=1}^{n} s_{k_{i j}}^{1} x_{j} \leq s_{t_{i}}^{1} \\
& \sum_{j=1}^{n} s_{k_{i j}}^{2} x_{j} \leq s_{t_{i}}^{2} \\
& \sum_{j=1}^{n} s_{k_{i j}}^{3} x_{j} \leq s_{t_{i}}^{3} \\
& \sum_{j=1}^{n} s_{k_{i j}}^{4} x_{j} \leq s_{t_{i}}^{4} \\
& \sum_{j=1}^{n} s_{a_{i j}}^{1} x_{j} \leq s_{b_{i}}^{1} \\
& \sum_{j=1}^{n} s_{a_{i j}}^{2} x_{j} \leq s_{b_{i}}^{2} \\
& \sum_{j=1}^{n} s_{a_{i j}}^{3} x_{j} \leq s_{b_{i}}^{3} \\
& \sum_{j=1}^{n} s_{a_{i j}}^{4} x_{j} \leq s_{b_{i}}^{4} \\
& x_{j} \geq 0 \\
& i \in \mathbb{N}, i=1, \ldots \ldots, m \\
& j \in \mathbb{N}, j=1, \ldots \ldots, n
\end{aligned}
$$

Step 4 : The problem can be solved as a classical linear programming problem. 


\subsection{Fuzzy objective function coefficients and fuzzy constraints}

Let us consider the following fuzzy linear programming problem with $m$ constraints and $n$ variables may formulate as follows:

$\max$

$$
\sum_{j=1}^{n} \tilde{c}_{j} x_{j}
$$

subject to

$$
\begin{gathered}
\sum_{j=1}^{n} \widetilde{k}_{i j} x_{j} \leq \tilde{t}_{i} \\
\sum_{j=1}^{n} \widetilde{a}_{i j} x_{j} \leq \widetilde{b}_{i} \\
x_{j} \geq 0
\end{gathered}
$$

where

$$
\begin{gathered}
i \in \mathbb{N}, i=1, \ldots \ldots, m \\
j \in \mathbb{N}, j=1, \ldots \ldots, n
\end{gathered}
$$

such that $\quad \tilde{\boldsymbol{c}}_{i j}=\left(\boldsymbol{c}_{i j}^{1}, \boldsymbol{c}_{i j}^{2}, \boldsymbol{c}_{i j}^{3}\right), \boldsymbol{\mu}_{c} ;\left(\overline{\boldsymbol{c}}_{i j}^{\mathbf{1}}, \overline{\boldsymbol{c}}_{i j}^{2}, \overline{\boldsymbol{c}}_{i j}^{3}\right), \boldsymbol{v}_{c}, \widetilde{\boldsymbol{k}}_{\boldsymbol{i} j}=$ $\left(\boldsymbol{k}_{i j}^{\mathbf{1}}, \boldsymbol{k}_{i j}^{2}, \boldsymbol{k}_{i j}^{3}\right), \boldsymbol{\mu}_{\boldsymbol{k}} ;\left(\overline{\boldsymbol{k}}_{i j}^{\mathbf{1}}, \overline{\boldsymbol{k}}_{i j}^{2}, \overline{\boldsymbol{k}}_{i j}^{3}\right), \boldsymbol{v}_{\boldsymbol{k}}$ and

$\tilde{\boldsymbol{t}}_{\boldsymbol{i}}=\left(\boldsymbol{t}_{i}^{\mathbf{1}}, \boldsymbol{t}_{i}^{2}, \boldsymbol{t}_{i}^{3}\right), \boldsymbol{\mu}_{\boldsymbol{t}} ;\left(\overline{\boldsymbol{t}}_{\boldsymbol{i}}^{\mathbf{1}}, \overline{\boldsymbol{t}}_{i}^{2}, \overline{\boldsymbol{t}}_{\boldsymbol{i}}^{3}\right), \boldsymbol{v}_{\boldsymbol{t}}$ are triangular intuitionistic fuzzy numbers TIFNs and $\boldsymbol{\mu}, \boldsymbol{v}$ are membership and and nonmembership functions respectively. $\widetilde{\boldsymbol{a}}_{\boldsymbol{i} j}=\left(\boldsymbol{a}_{i j}^{\mathbf{1}}, \boldsymbol{a}_{i j}^{\mathbf{2}}, \boldsymbol{a}_{i j}^{\mathbf{3}}\right), \widetilde{\boldsymbol{b}}_{\boldsymbol{i}}=\left(\boldsymbol{b}_{\boldsymbol{i}}^{\mathbf{1}}, \boldsymbol{b}_{\boldsymbol{i}}^{\mathbf{2}}, \boldsymbol{b}_{\boldsymbol{i}}^{\mathbf{3}}\right)$ are triangular fuzzy numbers TFNs. We use the following steps to solve this HFLPP.

Step 1 : The TIFNs $\tilde{\boldsymbol{c}}_{\boldsymbol{i} \boldsymbol{j}}, \widetilde{\boldsymbol{k}}_{\boldsymbol{i} \boldsymbol{j}}$ and $\tilde{\boldsymbol{t}}_{\boldsymbol{i}}$ transform to SFNs using equations (14-22) where $\boldsymbol{s h}\left(\tilde{c}_{i j}\right)=\left(\boldsymbol{s}_{\boldsymbol{c}_{i j}}^{\mathbf{1}}, \boldsymbol{s}_{\boldsymbol{c}_{i j}}^{2}, \boldsymbol{s}_{\boldsymbol{c}_{i j}}^{\mathbf{3}}, \boldsymbol{s}_{\boldsymbol{c}_{i j}}^{\mathbf{4}}\right)$, $\boldsymbol{s h}\left(\widetilde{k}_{i j}\right)=\left(s_{k_{i j}}^{1}, s_{k_{i j}}^{2}, s_{k_{i j}}^{3}, s_{k_{i j}}^{4}\right), \quad$ and $\quad \boldsymbol{s h}\left(\tilde{t}_{i}\right)=$ $\left(s_{t_{i}}^{1}, s_{t_{i}}^{2}, s_{t_{i}}^{3}, s_{t_{i}}^{4}\right)$.

Step 2 : The TFNs $\widetilde{\boldsymbol{a}}_{\boldsymbol{i} \boldsymbol{j}}$ and $\widetilde{\boldsymbol{b}}_{\boldsymbol{i}}$ transform to SFNs using equations (10 - 14) where $\boldsymbol{s h}\left(\widetilde{\boldsymbol{a}}_{i j}\right)=\left(\boldsymbol{s}_{\boldsymbol{a}_{i j}}^{1}, \boldsymbol{s}_{\boldsymbol{a}_{i j}}^{2}, \boldsymbol{s}_{\boldsymbol{a}_{i j}}^{\mathbf{3}}, \boldsymbol{s}_{\boldsymbol{a}_{i j}}^{\mathbf{4}}\right)$ and $\operatorname{sh}\left(\widetilde{b}_{i}\right)=\left(s_{b_{i}}^{1}, s_{b_{i}}^{2}, s_{b_{i}}^{3}, s_{b_{i}}^{4}\right)$.

Step 3 : The problem is reformulated as

$\operatorname{Max}$

$$
\sum_{j=1}^{n} s_{c_{i j}}^{t} x_{j}
$$

subject to

$$
\begin{gathered}
\sum_{j=1}^{n} s_{k_{i j}}^{1} x_{j} \leq s_{t_{i}}^{1} \\
\sum_{j=1}^{n} s_{k_{i j}}^{2} x_{j} \leq s_{t_{i}}^{2} \\
\sum_{j=1}^{n} s_{k_{i j}}^{3} x_{j} \leq s_{t_{i}}^{3} \\
\sum_{j=1}^{n} s_{k_{i j}}^{4} x_{j} \leq s_{t_{i}}^{4}
\end{gathered}
$$$$
\sum_{j=1}^{n} s_{a_{i j}}^{1} x_{j} \leq s_{b_{i}}^{1}
$$$$
\sum_{j=1}^{n} s_{a_{i j}}^{2} x_{j} \leq s_{b_{i}}^{2}
$$$$
\sum_{j=1}^{n} s_{a_{i j}}^{3} x_{j} \leq s_{b_{i}}^{3}
$$$$
\sum_{j=1}^{n} s_{a_{i j}}^{4} x_{j} \leq s_{b_{i}}^{4}
$$$$
\boldsymbol{x}_{j} \geq \mathbf{0}
$$

where

$$
\begin{gathered}
i \in \mathbb{N}, i=1, \ldots \ldots, m \\
j \in \mathbb{N}, j=1, \ldots \ldots, n \\
t \in \mathbb{N}, t=1,2,3,4
\end{gathered}
$$

Step 4 : The problem can be solved four times with four parameters of a SFN for the objective function coefficients using a classical linear programming problem.

Step 5 : The resulting maximum optimal solution of HFLPP is a SFN and we need to transform it to one crisp number. We proposed the following steps to obtain the maximum optimal solution:

Let maximum optimal solution $\boldsymbol{H}_{\boldsymbol{o p}}=\left(\boldsymbol{S}_{1}^{\boldsymbol{H}}, \boldsymbol{S}_{2}^{\boldsymbol{H}}, \boldsymbol{S}_{3}^{\boldsymbol{H}}, \boldsymbol{S}_{4}^{\boldsymbol{H}}\right)$ is a SFN that consists of three intervals $\boldsymbol{L}_{\boldsymbol{H}_{o p}}, \boldsymbol{C}_{\boldsymbol{H}_{o p}}, \boldsymbol{R}_{\boldsymbol{H}_{\text {op }}}$ where $\boldsymbol{L}_{\boldsymbol{H}_{o p}}=\left[\boldsymbol{S}_{1}^{H}, \boldsymbol{S}_{2}^{H}\right], \boldsymbol{C}_{\boldsymbol{H}_{\text {op }}}=\left[\boldsymbol{S}_{2}^{H}, \boldsymbol{S}_{3}^{H}\right], \boldsymbol{R}_{\boldsymbol{H}_{\text {op }}}=\left[\boldsymbol{S}_{3}^{H}, \boldsymbol{S}_{4}^{H}\right]$ as in Fig.

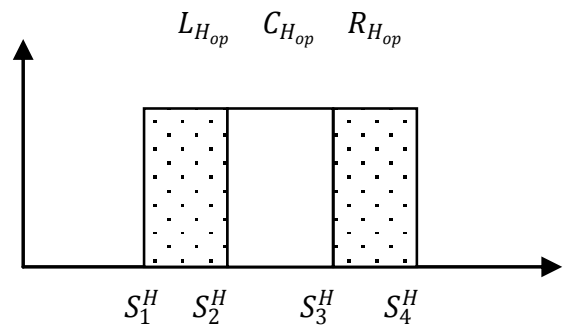

Fig. 4: Shadowed fuzzy number

We obtain the weights of the three intervals as follows:

$$
\begin{gathered}
w_{L}=\frac{S_{2}^{H}-S_{1}^{H}}{S_{4}^{H}-S_{1}^{H}} \\
w_{C}=\frac{S_{3}^{H}-S_{2}^{H}}{S_{4}^{H}-S_{1}^{H}} \\
w_{R}=\frac{S_{4}^{H}-S_{3}^{H}}{S_{4}^{H}-S_{1}^{H}}
\end{gathered}
$$

- The center values for the three intervals calculate as follows:

$$
C_{L}=\frac{S_{1}^{H}+S_{2}^{H}}{2}
$$




$$
\begin{gathered}
C_{C}=\frac{S_{2}^{H}+S_{3}^{H}}{2} \\
C_{R}=\frac{S_{3}^{H}+S_{4}^{H}}{2}
\end{gathered}
$$

- The value of the optimal solution $\boldsymbol{M}_{\boldsymbol{o p}}$ obtains as follows:

$$
M_{o p}=C_{L} \times w_{L}+C_{C} \times w_{C}+C_{R} \times w_{R}
$$

\subsection{Fuzzy objective function coefficients and Precise constraints}

Let us consider the following fuzzy linear programming problem with $m$ constraints and $n$ variables may formulate as follows:

$\max$

$$
\sum_{j=1}^{n} \tilde{c}_{j} x_{j}
$$

subject to

$$
\sum_{j=1}^{n} a_{i j} x_{j} \leq b_{i}
$$

$$
\boldsymbol{x}_{j} \geq \mathbf{0}
$$

where

$$
\begin{gathered}
i \in \mathbb{N}, i=1, \ldots \ldots, m \\
j \in \mathbb{N}, j=1, \ldots \ldots, n
\end{gathered}
$$

such that $\tilde{\boldsymbol{c}}_{\boldsymbol{i j}}=\left(\boldsymbol{c}_{\boldsymbol{i} \boldsymbol{j}}^{\mathbf{1}}, \boldsymbol{c}_{\boldsymbol{i j}}^{\mathbf{2}}, \boldsymbol{c}_{\boldsymbol{i j}}^{\mathbf{3}}\right), \boldsymbol{\mu}_{\boldsymbol{c}} ;\left(\overline{\boldsymbol{c}}_{\boldsymbol{i} \boldsymbol{i}}^{\mathbf{1}}, \overline{\boldsymbol{c}}_{\boldsymbol{i} \boldsymbol{j}}^{\mathbf{2}}, \overline{\boldsymbol{c}}_{\boldsymbol{i j}}^{\mathbf{3}}\right), \boldsymbol{v}_{\boldsymbol{c}}, \quad$ is a triangular intuitionistic fuzzy numbers TIFNs and $\boldsymbol{\mu}, \boldsymbol{v}$ are membership and non-membership functions respectively. We can also use type- 1 fuzzy number but the TIFN is used to describe steps of solving problem. $\boldsymbol{a}_{\boldsymbol{i} j}$ and $\boldsymbol{b}_{\boldsymbol{i}}$ are crisp numbers. The authors use the following steps to solve this HFLPP :

Step 1 : The TIFNs $\tilde{\boldsymbol{c}}_{\boldsymbol{i} \boldsymbol{j}}$ transform to a SFNs using equations $(14-22)$ where $\boldsymbol{s h}\left(\tilde{c}_{i j}\right)=\left(\boldsymbol{s}_{\boldsymbol{c}_{i j}}^{\mathbf{1}}, \boldsymbol{s}_{\boldsymbol{c}_{i j}}^{2}, \boldsymbol{s}_{\boldsymbol{c}_{i j}}^{\mathbf{3}}, \boldsymbol{s}_{\boldsymbol{c}_{i j}}^{\mathbf{4}}\right)$

Step 2 : The problem is reformulated as:

$$
\begin{aligned}
& \max \\
& \sum_{j=1}^{n} s_{c_{i j}}^{t} x_{j} \\
& \text { subject to } \\
& \sum_{j=1}^{n} a_{i j} x_{j} \leq b_{i} \\
& x_{j} \geq 0 \\
& i \in \mathbb{N}, i=1, \ldots \ldots, m \\
& j \in \mathbb{N}, j=1, \ldots \ldots, n
\end{aligned}
$$

Step 3 : The problem can be solved four times with four parameters of a SFN of for the objective function coefficients using a classical linear programming problem.
Step 4 : The resulting maximum optimal solution of HFLPP is a SFN and transforms to one crisp number using equations $(32-38)$

\section{ILLUSTRATIVE EXAMPLES Example 1}

We use the adaptive example as mentioned in [1] where the parameters of the constraints are only type- 1 fuzzy numbers. In these examples, TIFNs and TFNs are used to represent the constraints coefficients and right hand side values of the constraints. The problem is solved using steps in section 4.1. Consider the following HFLPP:

$$
\begin{array}{lc}
\max & \boldsymbol{z}=\mathbf{5} \boldsymbol{x}_{\mathbf{1}}+\mathbf{4} \boldsymbol{x}_{\mathbf{2}} \\
\text { subject to } & \widetilde{\boldsymbol{k}}_{\mathbf{1 1}} \boldsymbol{x}_{\mathbf{1}}+\widetilde{\boldsymbol{k}}_{\mathbf{1 2}} \boldsymbol{x}_{\mathbf{2}} \leq \tilde{\boldsymbol{t}}_{\mathbf{1}} \\
& \widetilde{\boldsymbol{a}}_{\mathbf{1 1}} \boldsymbol{x}_{\mathbf{1}}+\widetilde{\boldsymbol{a}}_{\mathbf{1 2}} \boldsymbol{x}_{\mathbf{2}} \leq \widetilde{\boldsymbol{b}}_{\mathbf{1}} \\
& \boldsymbol{x}_{\mathbf{1}}, \boldsymbol{x}_{\mathbf{2}} \geq \mathbf{0}
\end{array}
$$

where $\widetilde{\boldsymbol{k}}_{\mathbf{1 1}}=(3,4,5),(2,4,6) ; 1,0, \widetilde{\boldsymbol{k}}_{\mathbf{1 2}}=(4,5,6),(2,5,7)$ 1,0 and $\tilde{\boldsymbol{t}}_{\mathbf{1}}=(20,24,30),(18,24,32) ; 1,0$. Also, $\widetilde{\boldsymbol{a}}_{\mathbf{1 1}}=(3,4$, $6), \widetilde{\boldsymbol{a}}_{12}=(0.5,1,2)$ and $\widetilde{\boldsymbol{b}}_{1}=(6,12,15)$ are three TFNs. We transform the TIFNs to SFNs and the results are as the following:

$$
\begin{aligned}
& \boldsymbol{s h}\left(\widetilde{\boldsymbol{k}}_{\mathbf{1 1}}\right)=(2.76,3.35,4.65,5.24), \\
& \boldsymbol{s h}\left(\widetilde{\boldsymbol{k}}_{\mathbf{1 2}}\right)=(4.12,4.2,5.66,6.25), \\
& \boldsymbol{s h}\left(\tilde{\boldsymbol{t}}_{\mathbf{1 2}}\right)=(19.43,21.98,26.85,30.54) .
\end{aligned}
$$

Also, we transform the TFNs to SFNs as the following

$$
\begin{aligned}
& \boldsymbol{s h}\left(\widetilde{\boldsymbol{a}}_{11}\right)=(3.06,3.56,4.89,5.89), \\
& \boldsymbol{s h}\left(\widetilde{\boldsymbol{a}}_{12}\right)=(0.52,0.77,1.46,1.96), \\
& \boldsymbol{s h}\left(\widetilde{\boldsymbol{b}}_{\mathbf{1}}\right)=(6.5,9.5,13.25,14.75) .
\end{aligned}
$$

The problem can be reformulated to be as the following:

$$
\max \quad z=5 x_{1}+4 x_{2}
$$

subject to

$$
2.76 x_{1}+4.12 x_{2} \leq 19.43
$$$$
3.35 x_{1}+4.2 x_{2} \leq 21.98
$$$$
4.65 x_{1}+5.66 x_{2} \leq 26.85
$$$$
5.24 x_{1}+6.25 x_{2} \leq 30.54
$$$$
3.06 x_{1}+0.52 x_{2} \leq 6.5
$$$$
3.56 x_{1}+0.77 x_{2} \leq 9.5
$$$$
4.89 x_{1}+1.46 x_{2} \leq 13.25
$$$$
5.89 x_{1}+1.96 x_{2} \leq 14.75
$$

$$
x_{1}, x_{2} \geq 0
$$

Then we apply the classical linear programming steps using Matlab tool to obtain $x_{1}=1.27, x_{2}=3.7$ and $z=21.16$. While the original problem used triangular fuzzy numbers only and its results were $x_{1}=1.5, x_{2}=3$ and $z=19.5$ 
[1]. We find that, the results of the new approach are approximately equivalent the results of the original problem.

\section{Example 2}

This example is a modified version from [13] where TIFNs only are used to represent objective function coefficients, constraints coefficients and right hand side values. We use TIFNs, TFNs and Gaussian fuzzy numbers GFNs to represent objective function parameters and constraints parameters. The problem is solved using the steps in section 4.2. Consider the following HFLPP:

$$
\begin{array}{lc}
\max & z=\widetilde{\mathbf{5}} \boldsymbol{x}_{1}+\widetilde{\mathbf{3}} \boldsymbol{x}_{2} \\
\text { subject to } & \widetilde{\mathbf{4}} \boldsymbol{x}_{1}+\widetilde{\mathbf{3}} \boldsymbol{x}_{2} \leq \widetilde{\mathbf{1 2}} \\
& \widetilde{\mathbf{1}} \boldsymbol{x}_{1}+\overline{\mathbf{3}} \boldsymbol{x}_{2} \leq \widetilde{\mathbf{6}} \\
& \boldsymbol{x}_{1}, \boldsymbol{x}_{2} \geq \mathbf{0}
\end{array}
$$

where $\widetilde{\mathbf{5}}=(4,5,6),(4,5,6.1) ; 1,0$ and $\widetilde{\mathbf{3}}=(2.5,3,3.2),(2$, $3,3.5) ; 1,0$ are two TIFNs. $\widetilde{\mathbf{4}}=(3,4,5), \widetilde{\mathbf{3}}=(2.3,3,4)$, are two TFNs. $\widetilde{\mathbf{1}}=(\boldsymbol{m}=\mathbf{1} ; \boldsymbol{\sigma}=\mathbf{0 . 4})$ and $\widetilde{\mathbf{3}}=(\boldsymbol{m}=\mathbf{3} ; \boldsymbol{\sigma}=$ 0.2) are two GFNs. Also, $\widetilde{\mathbf{1 2}}=(11,12,13),(11,12,14) ; 1$, 0 and $\widetilde{\mathbf{6}}=(5.5,6,7.5),(5,6,8.1) ; 1,0$ are two TIFNs. We transform the TIFNs to SFNs and the results are the following:

$\boldsymbol{s h}(\widetilde{\mathbf{5}})=(4.06,4.55,5.48,5.98)$,

$\boldsymbol{s h}(\widetilde{\mathbf{3}})=(2.18,2.65,3.16,3.6)$,

$\boldsymbol{s h}(\widetilde{\mathbf{1 2}})=(11.05,11.54,12.65,13.12)$,

$\boldsymbol{s h}(\widetilde{\mathbf{6}})=(5.21,5.68,6.81,7.3)$.

Also, we transform TFNs to SFNs as the following:

$\boldsymbol{s h}(\widetilde{\mathbf{4}})=(3.04,3.54,4.46,4.96)$,

$\boldsymbol{s h}(\widetilde{\mathbf{3}})=(2.33,2.68,3.46,3.96)$,

$\boldsymbol{s h}(\widetilde{\mathbf{1}})=(0.36,0.66,1.34,1.64)$,

$\boldsymbol{s h}(\widetilde{3})=(2.68,2.83,3.17,3.32)$.

The problem can be reformulated as the following:

$$
\max \quad z=\operatorname{sh}(\widetilde{5}) x_{1}+\operatorname{sh}(\widetilde{3}) x_{2}
$$

subject to

$$
\begin{gathered}
3.04 x_{1}+2.33 x_{2} \leq 11.05 \\
3.54 x_{1}+2.68 x_{2} \leq 11.54 \\
4.46 x_{1}+3.46 x_{2} \leq 12.65 \\
4.96 x_{1}+3.96 x_{2} \leq 13.12 \\
0.36 x_{1}+2.68 x_{2} \leq 5.21 \\
0.66 x_{1}+2.83 x_{2} \leq 5.68 \\
1.34 x_{1}+3.17 x_{2} \leq 6.81 \\
1.64 x_{1}+3.32 x_{2} \leq 7.3
\end{gathered}
$$

$$
x_{1}, x_{2} \geq 0
$$

\begin{tabular}{|c|c|c|c|c|}
\hline $\begin{array}{l}\text { Product } \\
\text { type }\end{array}$ & $\begin{array}{c}\text { Process } \\
1 \\
\text { hours }\end{array}$ & $\begin{array}{c}\text { Process } \\
2 \\
\text { hours }\end{array}$ & $\begin{array}{c}\text { Process } \\
\mathbf{3} \\
\text { hours }\end{array}$ & $\begin{array}{c}\text { Profit } \\
\text { hundred } \\
\$\end{array}$ \\
\hline Automobile & 15 & 24 & 21 & 25 \\
\hline Truck & 30 & 6 & 14 & 48 \\
\hline Total hours & 45000 & 24000 & 28000 & \\
\hline
\end{tabular}

The resulting optimal value is $\mathrm{SFN}=(10.74,12.04,14.5$, 15.82), $\boldsymbol{x}_{\mathbf{1}}=2.65$ and $\boldsymbol{x}_{2}=0$. We calculate the crisp optimal value where $\boldsymbol{M}_{\text {op }}=13.28$.

\section{Example 3}

This example is a modified version from [13] and [24] where TIFNs in [13] and interval-valued fuzzy numbers in [24] are used to represent the objective function coefficients. The TIFNs are used to represent parameters of the objective function. The problem is solved using steps in section 4.3. Let a factory produces automobiles and truck where each product need three processes. The production conditions are given in Table 1.

Table 1: Production conditions

Consider the following HFLPP:

$$
\max \quad z=\widetilde{\mathbf{2 5}} \boldsymbol{x}_{1}+\widetilde{\mathbf{4 8}} \boldsymbol{x}_{2}
$$

subject to

$$
\begin{gathered}
15 x_{1}+30 x_{2} \leq 45000 \\
24 x_{1}+6 x_{2} \leq 24000 \\
21 x_{1}+14 x_{2} \leq 28000 \\
x_{1}, x_{2} \geq 0
\end{gathered}
$$

where $\widetilde{\mathbf{2 5}}=(19,25,33),(18,25,34) ; 1,0$ and $\widetilde{\mathbf{4 8}}=(44,48$, 54), (43, 48, 56); 1,0 are two TIFNs. The constraints parameters are crisp numbers. We transform the TIFNs to SFNs and the results are as the following:

$\boldsymbol{s h}(\widetilde{\mathbf{2 5}})=(21.87,22.37,28.44,28.94)$,

$\boldsymbol{s h}(\widetilde{\mathbf{4 8}})=(45.66,46.16,50.86,51.36)$.

The problem can be reformulated as the following:

$$
\begin{gathered}
\max \quad z=\operatorname{sh}(\widetilde{25}) x_{1}+\operatorname{sh}(\widetilde{48}) x_{2} \\
15 x_{1}+30 x_{2} \leq 45000 \\
24 x_{1}+6 x_{2} \leq 24000 \\
21 x_{1}+14 x_{2} \leq 28000 \\
x_{1}, x_{2} \geq 0
\end{gathered}
$$

The resulting optimal value is the $\mathrm{SFN}=(68490,69240$, $77795,78670), \boldsymbol{x}_{\mathbf{1}}=500, \boldsymbol{x}_{\mathbf{2}}=1250$. We calculated the crisp optimal value where $\boldsymbol{M}_{\boldsymbol{o p}}=73587$. In the original problem [24], $\mathbf{x}_{1}=500, \mathbf{x}_{2}=1250$ and the optimal solution $=$ 73104.7. 


\section{Remark 1}

When solving the problem in example 3 using a new approach, the values of objective function variables are $\left(\boldsymbol{x}_{\mathbf{1}}=\right.$ $\left.0, \boldsymbol{x}_{2}=1500\right)$ with SFN parameters $(68490,69240)$ and $\left(\boldsymbol{x}_{1}=\right.$ $\left.500, x_{2}=1250\right)$ with SFN parameters $(77795,78670)$. We select solution that more near to optimal crisp solution where $\boldsymbol{M}_{\text {op }}=73587$.

In [13], the optimal solution for example 3 is $\left(x_{1}=0, x_{2}=\right.$ $1500)$ with optimal objective value 70500 and $\left(x_{1}=0, x_{2}=\right.$ 1500 ) with optimal objective value 62000 .

\subsection{Discussions}

Based on the previous results, the following remarks are found:

1. The new approach can handle type-1 fuzzy numbers and higher type of fuzzy numbers i.e. intuitionistic fuzzy numbers. This feature provides more flexibility for representing linear programming problems.

2. The new approach can use different types of membership functions in one problem as in example 2 which TFNs and GFNs are used.

3. The new approach satisfies all the constraints including non-negative constraint.

4. Solving the same illustrative examples as in [1] and [24], using the newly proposed approach results in approximately the same optimal solution. This proves the validity of the proposed approach.

\subsection{Advantage of the proposed method}

The basic improvement in the new approach against previous approaches, it can handle different types of fuzzy numbers and also higher type like IFNs in the same problem with different cases of FLPP. This advantage provides more flexibility for solving FLPPs.

\section{CONCLUSION}

The hybrid fuzzy-linear programming problem is characterized by addressing different types of uncertain numbers that represent parameters values of problem. In this paper, we propose a new approach to solve this kind of problems using an improved form of shadowed fuzzy numbers. The proposed approach includes getting SFN from type-1 fuzzy numbers and intuitionistic fuzzy numbers which preserving the characteristics of uncertainty for them. The SFNs used in hybrid fuzzy-linear programming example to unify the form of parameters and in the same time simplify calculation operations. The newly proposed approach results are the same as the optimization results of the original example which prove the validity of the new approach. This new technique provides the ability to treat with more hybrid uncertain numbers for the future works in solving FLPPs.

\section{REFERENCES}

[1] George. J. Klir, Bo. Yuan, (1995) Fuzzy Sets and Fuzzy Logic Theory and Applications. Prentice Hall press.

[2] George J. Klir, Wierman Mark 1, (1999 ) Uncertainty Based Information Elements of Generalized Information Theory. Springer-Verlag Berlin Heidelberg GmbH .

[3] Atanassov .K, (1999) Intuitionistic Fuzzy Sets. Theory and Applications. Physica-Verlag, Heidelberg New York.
[4] Atanassov .K, (1986) Intuitionistic fuzzy sets. Fuzzy Sets and Systems Vol. 20, Issue (1), pp.87 -96.

[5] Witold Pedrycz, (1998) Shadowed Sets: Representing and Processing Fuzzy Sets. IEEE Transactions on systems, man, and cybernetics-part B: Cybernetics, Vol. 28, No. 1 .

[6] Pedrycz Witold. (2005). Granular Computing with Shadowed Sets. Ślęzak et al. (Eds.): RSFDGrC 2005, LNAI 3641, Springer-Verlag Berlin Heidelberg, pp. 23 -32 .

[7] Mohamed A. H. El_Hawy, Hesham A. Hassan, Hesham A. Hefny, Khaled T. Wassif , (May 2015) An Improved Fuzzy Number Approximation using Shadowed Sets. International Journal of Computer Applications (0975 8887), Vol. 118 , No.25, pp. 9-15.

[8] Mohamed A. H. El_Hawy, Hesham A. Hassan, Hesham A. Hefny, Khaled T. Wassif (2015) A Proposed Shadowed Intuitionistic Fuzzy Numbers. Computer Engineering \& Systems (ICCES), $201510^{\text {th }}$, IEEE.

[9] R.E. Bellmann, L.A. Zadeh, (1970 ) Decision making in fuzzy environment. Management Sci. Vol.17, pp. 141164.

[10] Jagdeep Kaur, Amit Kumar, (2016) An Introduction to Fuzzy Linear Programming Problems Theory, Methods and Applications. Springer International Publishing Switzerland

[11] P.P. Angelov, (1997) Optimization in an intuitionistic fuzzy environment. Fuzzy sets and systems. Vol.86, pp. 299-306.

[12] Jaroslav Ramík, Milan Vlach, (2016 ) Intuitionistic fuzzy linear programming and duality: a level sets approach Fuzzy Optimization and Decision Making, pp. 1-33, Springer Science Business Media New York.

[13] Dipti Dubey, Aparna Mehra, "Linear programming with Triangular Intuitionistic Fuzzy Number", EUSFLATLFA, Aix-les-Bains, France, 2011.

[14] D. Dubeyetal, (2012) Fuzzy linear programming under interval uncertainty based on IFS representation. Fuzzy Sets and Systems, Vol.188, pp. $68-87$.

[15] A. Kaufmann, M.M. Gupta, (1985) Introduction to Fuzzy Arithmetic Theory and Applications. Van Nostrand Reinhold, New York.

[16] C. R. Bector, Suresh Chandra, ( 2005) Fuzzy Mathematical Programming and Fuzzy Matrix Games. Springer-Verlag Berlin Heidelberg.

[17] Grzegorzewski, P, (2003) Distances and orderings in a family of intuitionistic fuzzy numbers. In: EUSFLAT Conf., pp. 223-227.

[18] M. Kumar and S.P. Yadav, (2012 ) Analyzing Fuzzy System Reliability Using Arithmetic Operations on Different Types of Intuitionistic Fuzzy Numbers. K. Deep et al. (Eds.): Proceedings of the International Conference on SocProS 2011, AISC 130, pp. 725-736. Springer India.

[19] Witold Pedrycz, (2009) From Fuzzy Sets to Shadowed Sets: Interpretation and Computing. INTERNATIONAL JOURNAL OF INTELLIGENT SYSTEMS, VOL. 24 , pp. 48-61. 
[20] Olgierd Hryniewicz, (2006) An Evaluation of the Reliability of Complex Systems Using Shadowed Sets and Fuzzy Lifetime Data. International Journal of Automation and Computing, Vol. 2 ,pp. 145-150.

[21] Tahayori, H.; Sadeghian, A.; Pedrycz W, (2013) Induction of Shadowed Sets Based on the Gradual Grade of Fuzziness Fuzzy Systems, IEEE Transactions on ,Vol. 21, 5, pp.937-949.

[22] Xia Liang , Cuiping Wei, Meimei Xia, (September, 2013) New Entropy, Similarity Measure of Intuitionistic Fuzzy Sets and their Applications in Group Decision
Making. International Journal of Computational Intelligence Systems, Vol. 6, No. 5, pp. 987-1001.

[23] Mohamed. A. H. El-Hawy, K. T. Wassif, H. A. Hefny and H. A. Hassan, (Dec. 2015) Hybrid multi-attribute decision making based on shadowed fuzzy numbers. IEEE Seventh International Conference on Intelligent Computing and Information Systems (ICICIS), Cairo, 2015, pp. 514-521.

[24] Jin-Shieh Su, (2007) Fuzzy Programming Based on Interval-Valued Fuzzy Numbers and Ranking. Int. J. Contemp. Math. Sciences, Vol. 2, No. 8, pp. $393-410$. 